\title{
Crescimento, esporulação e viabilidade de fungos entomopatogênicos em meios contendo diferentes concentrações do óleo de Nim (Azadirachta indica)
}

\author{
Growth, esporulation and viability of entomopathogenic fungi under mediums \\ with differents Nim oil (Azadirachta indica) concentrations
}

\section{Renata Paro Marques $^{1}$ Antonio Carlos Monteiro ${ }^{2}$ Gener Tadeu Pereira ${ }^{3}$}

\section{RESUMO}

A associação de extratos de origem vegetal com fungos entomopatogênicos pode aumentar a eficiência do controle biológico de pragas, reduzir custos e impactos ambientais. No presente trabalho, avaliou-se, através da concentração inibitória mínima, o efeito do óleo de nim (NIM-IGO) sobre o crescimento, esporulação e viabilidade de Metarhizium anisopliae, Beauveria bassiana e Paecilomyces farinosus. Utilizou-se o meio BDA, contendo diferentes concentrações de óleo de nim (C1: $5 \%$ de óleo de nim, e sucessivamente concentrações iguais a $1 / 2$ da concentração anterior, até C11: 0,0048\% de óleo de nim). O óleo de nim reduziu o crescimento de colônias de B. bassiana e P. farinosus, que não diferiram significativamente do controle apenas na concentração C11, mas para M. anisopliae o mesmo efeito foi observado com 0,039\% de óleo de nim (C8). A esporulação também foi significativamente reduzida pelo óleo de nim, exceto na concentração $C 11$ para B. bassiana; contudo, não se verificou efeito do óleo na viabilidade de esporos dos fungos.

Palavras-chave: Beauveria bassiana, Metarhizium anisopliae, Paecilomyces farinosus, compatibilidade controle biológico, controle microbiano.

\section{ABSTRACT}

The association of vegetable extracts with entomopathogenic fungi can increase the efficiency of biological control of pests, to reduce costs and environmental impacts. The present work aimed to evaluate, through minimal inibitory concentration, the effect of the neem oil (NIM-I-GO) on the growth, esporulation and viability of Metarhizium anisopliae, Beauveria bassiana and Paecilomyces farinosus. For this purpose the PDA medium was used containing different concentrations of neem oil
(C1: $5 \%$ of neem oil, and successively concentrations $1 / 2$ of the previous concentration, until the concentration C11:0,0048\% of neen oil). The neen oil reduced the growth of $\boldsymbol{B}$. bassiana and $\boldsymbol{P}$. farinosus, which didn't differ significantly of the control at the concentration C11. Similar effect was observed for M. anisopliae with 0,039\% of neem oil (C8). The esporulation was also significantly reduced by the neem oil, except at the concentration C11 for B. bassiana; however, no oil effect was observed on the viability of the fungi spores.

Key words: Beauveria bassiana, Metarhizium anisopliae, Paecilomyces farinosus, biological control, compatibility, microbial control.

\section{INTRODUÇÃO}

O uso de compostos químicos sintéticos tem sido, há muitos anos, o principal meio de controle de pragas. Apesar de sua significativa contribuição para a produção agrícola, o uso intensivo e indiscriminado destes produtos favoreceu o surgimento de pragas secundárias e não conseguiu eliminar os problemas já existentes; além disso, são altamente tóxicos, sendo prejudiciais ao ambiente e à saúde humana.

Por serem de fácil obtenção e utilização, terem baixo custo e minimizarem os problemas apresentados pelos produtos químicos sintéticos, os inseticidas naturais de origem vegetal podem vir a se constituir em importantes agentes de controle. Extratos biologicamente ativos obtidos de folhas, frutos,

${ }^{1}$ Biomédica, Mestre, Ex-aluna do Programa de Pós-graduação em Microbiologia, Faculdade de Ciências Agrárias e Veterinárias (FCAV), Universidade Estadual Paulista (UNESP). E-mail:prontolab.colina@itelefonica.com.br

${ }^{2}$ Biólogo, Departamento de Produção Vegetal, FCAV, UNESP. Via de Acesso Professor Paulo Donato Castellane, s/n, 14884-900,

Jaboticabal, SP. E-mail: montecar@fcav.unesp.br. Autor para correspondência.

${ }^{3}$ Estatístico, Departamento de Ciências Exatas, FCAV, UNESP. E-mail: genertp@fcav.unesp.br 
sementes e tronco de nim [Azadirachta indica (A.) Juss.] são reconhecidos por suas propriedades inseticidas, sendo a azadiractina considerada o composto ativo mais potente da árvore de nim, isolada por BUTTERWORTH \& MORGAN (1968)

Outro recurso é o controle biológico com a utilização de fungos entomopatogênicos, também uma alternativa viável devido à facilidade de produção, aplicação e eficácia. Podem ser usados isoladamente ou integrados com outros métodos, como os inseticidas naturais de origem vegetal, feromônios, variedades de plantas resistentes a insetos, etc (LOURENÇÃO et al., 1993). A associação destes com extratos de nim para o controle de pragas é uma possibilidade recente, podendo resultar em sinergismo entre os dois, uma aproximação considerada promissora, mas ainda pouco estudada.

Nessa linha de pesquisa, SHASHI et al. (1998) avaliaram o efeito de altas concentrações de azadiractina sobre o fungo Beauveria brongniartii (Sacc.) Petch , sem contudo obterem resultados significativos. PRAMILA et al. (1999) verificaram que sete produtos comerciais obtidos de nim, usados nas concentrações de 10.000, 1.000 e 100ppm, tiveram efeitos diversos no crescimento de Beauveria bassiana (Bals.) Vuill., variando de inibitórios a estimulantes. LANDA \& BOHATA (1999) constataram que não houve efeito negativo no crescimento e germinação de conídios do fungo entomopatogênico Paecilomyces fumosoroseus (Wise) Brown \& Smith, quando estudaram sua compatibilidade com dois inseticidas derivados de nim.

Vários trabalhos referem-se ao efeito de derivados do nim como inibidores do crescimento de fungos (CHATTOPADHYAY, 1999; STEINHAUER, 1999; AMADIOHA, 2000); contudo, pouco se conhece da ação de extratos de nim sobre fungos entomopatogênicos.

Assim, este trabalho teve como objetivo avaliar, em laboratório, a ação do óleo de nim sobre fungos entomopatogênicos, visando à possibilidade do uso associado destes no controle biológico de insetos.

\section{MATERIAL E MÉTODOS}

Utilizram-se os isolados JAB 07 de Beauveria bassiana (obtido de Musca domestica), E9 de Metarhizium anisopliae (Metsch.) Sorokin (obtido de Deois flavopicta) e CG 195 de Paecilomyces farinosus (Hom ex SF Gray) Brown \& Smith (obtido de pupa de Chlosyne lacinia), cultivados em placas de Petri contendo meio de batata- dextrose-ágar (BDA), acondicionadas em estufa a 27 $\pm 1^{\circ} \mathrm{C}$, em ausência de luz, durante 15 dias.

Como inseticida natural de origem vegetal utilizou-se um produto comercial de óleo de nim emulsionado, marca NIM-I-GO, contendo 1.200ppm de azadiractina (comunicação verbal do responsável pela marca comercial, 2003) ${ }^{1}$.

$\mathrm{O}$ efeito do óleo de nim sobre os fungos entomopatogênicos foi avaliado através da concentração inibitória mínima, segundo metodologia descrita por BAKER (1970). Em um frasco contendo meio $\mathrm{BDA}$ estéril, à temperatura de 45 a $50^{\circ} \mathrm{C}$, adicionou-se óleo de nim necessário para se obter a concentração de 5\% no meio de cultura. Após vigorosa agitação, mantendo-se a temperatura do meio, verteuse metade do conteúdo do frasco em placas de Petri $\left(20 \mathrm{~mL} \mathrm{placa}^{-1}\right)$. A outra metade verteu-se em outro frasco com igual quantidade de meio, nas mesmas condições, obtendo-se neste segundo frasco, metade da concentração de óleo de nim do frasco inicial. Em seguida, repetiu-se o procedimento sucessivamente, obtendo-se ao final, placas de Petri contendo BDA com as seguintes concentrações: $\mathrm{C} 1: 5 \%$ de óleo de $\operatorname{nim}\left(6 \times 10^{-5} \mathrm{~mL}\right.$ de azadiractina $\mathrm{mL}$ de meio $\left.{ }^{-1}\right) ; \mathrm{C} 2$ : $2,5 \%$ de óleo de nim $\left(3 \times 10^{-5} \mathrm{~mL}\right.$ de azadiractina $\mathrm{mL}^{-1}$ de meio); C3: 1,25\% de óleo de nim (1,5 x 10 $0^{-5} \mathrm{~mL}$ de azadiractina $\mathrm{mL}$ de meio $^{-1}$ ); $\mathrm{C} 4: 0,625 \%$ de óleo de $\operatorname{Nim}\left(0,75 \times 10^{-5} \mathrm{~mL}\right.$ de azadiractina $\mathrm{mL}$ de meio $\left.^{-1}\right)$; C5: $0,312 \%$ de óleo de nim $\left(0,37 \times 10^{-5} \mathrm{~mL}\right.$ de azadiractina $\mathrm{mL}$ de meio $\left.^{-1}\right)$; C6: $0,156 \%$ de óleo de $\operatorname{nim}\left(0,187 \times 10^{-5} \mathrm{~mL}\right.$ de azadiractina $\mathrm{mL}$ de meio $\left.^{-1}\right)$; C7: $0,078 \%$ de óleo de nim $\left(0,093 \times 10^{-5} \mathrm{~mL}\right.$ de azadiractina $\mathrm{mL}$ de meio $^{-1}$ ); C8: 0,039\% de óleo de $\operatorname{nim}\left(0,047 \times 10^{-5} \mathrm{~mL}\right.$ de azadiractina $\mathrm{mL}$ de meio $\left.^{-1}\right)$; C9: $0,019 \%$ de óleo de nim $\left(0,023 \times 10^{-5} \mathrm{~mL}\right.$ de azadiractina $\mathrm{mL}$ de meio $^{-1}$ ); C10: 0,0097\% de óleo de $\operatorname{nim}\left(0,012 \times 10^{-5} \mathrm{~mL}\right.$ de azadiractina $\mathrm{mL}$ de meio $\left.^{-1}\right)$; C11: $0,0048 \%$ de óleo de nim $\left(0,006 \times 10^{-5} \mathrm{~mL}\right.$ de azadiractina $\mathrm{mL}$ de $\left.\mathrm{meio}^{-1}\right)$. Preparou-se ainda placas contendo apenas o meio BDA (controle).

Estas placas, que constituíram os tratamentos do ensaio, foram inoculadas, por picada central, a partir de suspensões dos isolados dos fungos, com a concentração de $1 \times 10^{7}$ conídios $/ \mathrm{mL}$. Em seguida, foram acondicionadas em estufa à $27 \pm 1^{\circ} \mathrm{C}$, em ausência de luz, durante 15 dias.

O crescimento radial das colônias, a produção e viabilidade de conídios foram utilizados como parâmetros de avaliação do desempenho dos fungos em relação às diferentes quantidades de óleo de nim adicionadas ao meio de cultura.

O crescimento da colônia foi quantificado através de medidas, em milímetros, de dois diâmetros 
previamente marcados na parte externa do fundo da placa de Petri. As medidas foram efetuadas a cada três dias, do 3a ao $15^{\circ}$ dia após a inoculação. Cada placa correspondeu a uma repetição, e para cada concentração de óleo de nim (tratamento) fez-se 5 placas.

A produção de conídios foi avaliada coletando-se em cada colônia uma amostra central, uma intermediária e uma periférica, com o auxílio de um anel metálico esterilizado de $8 \mathrm{~mm}$ de diâmetro, no $15^{\circ}$ dia de incubação. Para cada tratamento, coletaram-se amostras de 3 colônias. Estas amostras foram transferidas individualmente para tubos de ensaio contendo $10 \mathrm{~mL}$ de uma mistura $1: 1$ de solução de $\mathrm{NaCl}(0,89 \%, \mathrm{p} / \mathrm{v})$ e Tween $80^{\circledR}(0,1 \%, \mathrm{v} / \mathrm{v})$ esterilizada. Após remoção dos conídios por vigorosa agitação em agitador elétrico de tubos, foi feita a contagem ao microscópio com 400 aumentos, em câmara de Neubauer, utilizando-se diluição da suspensão quando necessário. A partir dos valores médios das amostras colhidas em cada colônia, calculou-se a produção média de conídios em toda a colônia, com base nas medidas de diâmetro efetuadas para avaliação do crescimento radial das mesmas.

Nos ensaios com M. anisopliae, utilizaramse meios de cultura contendo óleo de nim até a C9, porque, em pré-ensaios realizados, os diâmetros das colônias nesta concentração não diferiram do controle. Nos ensaios com B. bassiana e P. farinosus, utilizaram-se meios contendo óleo de nim até a C11.

A viabilidade dos conídios foi avaliada, através de microcultivo e exame direto em lâmina, utilizando-se duas situações: a) na primeira, lâminas de microscopia esterilizadas foram recobertas com uma fina camada de BDA contendo, cada lâmina, uma das concentrações do óleo de nim já mencionadas. Em seguida, foram inoculadas com suspensões de conídios obtidas de colônias crescidas em BDA, isento de óleo de nim; b) na segunda situação, lâminas de microscopia esterilizadas foram recobertas com uma fina camada de BDA, isento de óleo de nim. A inoculação foi realizada com suspensões de conídios obtidos de colônias cultivadas em BDA contendo uma das diferentes concentrações de óleo de nim já mencionadas.

Para a realização dos ensaios, foram previamente marcados três círculos na face inferior de cada lâmina. Sobre o meio de cultura foi inoculado, em cada círculo, $0,1 \mathrm{~mL}$ de suspensão fúngica com $10^{5}$ conídios $\mathrm{mL}^{-1}$. As lâminas, acondicionadas individualmente em placas de Petri com algodão umidecido, esterilizadas, foram então incubadas a 27 $\pm 1^{\circ} \mathrm{C}$, em ausência de luz, durante $15 \mathrm{~h}$. Após este período, foi colocada uma gota do corante lactofenol azul-de-algodão sobre a área do círculo, para deter a germinação e facilitar a visualização do conídio. Em seguida, foi feita a observação de 150 conídios em cada área da lâmina, utilizando-se microscópio com 400 aumentos. Foram contados conídios germinados e não germinados, estabelecendo-se depois a porcentagem de germinação. Cada lâmina correspondeu a uma repetição e para cada tratamento fez-se três lâminas.

Os ensaios foram conduzidos segundo o delineamento inteiramente casualizado. Para a avaliação do crescimento, os dados foram analisados por meio de regressão linear, seguida de teste de Paralelismo (teste t) e Coincidência (teste F) entre as retas (DIXON \& MASSEY JUNIOR, 1969). Para a produção e viabilidade de esporos, os dados foram submetidos à análise de variância pelo teste $\mathrm{F}$ e as médias comparadas pelo teste de Tukey, a 5\% de probabilidade, pelo programa ESTAT.

\section{RESULTADOS E DISCUSSÃO}

O crescimento radial das colônias de $\boldsymbol{B}$. bassiana e $\boldsymbol{P}$. farinosus não foi afetado pelo óleo de nim na concentração C 11, que não diferiu significativamente do controle, mostrando que a taxa de crescimento para ambas as concentrações foi estatisticamente a mesma. Nas demais concentrações o óleo inibiu o crescimento do fungo (Figura 1, B e C).

Com relação a $M$. anisopliae obteve-se um resultado distinto do encontrado para os outros dois fungos, pois, a partir da concentração C8, o óleo de nim não teve efeito sobre o crescimento, indicando que este fungo é menos sensível à ação do óleo de nim (Figura 1A).

A esporulação de $\boldsymbol{B}$. bassiana, na concentração C11, não foi influenciada pelo óleo de nim. Nas demais concentrações, assim como em todas as concentrações para $\boldsymbol{P}$. farinosus e $\boldsymbol{M}$. anisopliae, houve efeito significativo do óleo de nim, reduzindo crescentemente a produção de esporos com o aumento das concentrações. Entretanto, para M. anisopliae, como a produção de esporos só foi avaliada até C9, é possível presumir que, em diluições subsequentes, próximas a esta, não ocorra efeito significativo do óleo de nim sobre a esporulação do fungo (Tabela 1).

A germinação dos conídios dos três fungos estudados não foi afetada pelas diferentes concentrações de óleo de nim, considerando-se tanto a situação em que os esporos foram colhidos de colônias cultivadas no meio contendo as diferentes concentrações de óleo de nim (Tabela 2), como 


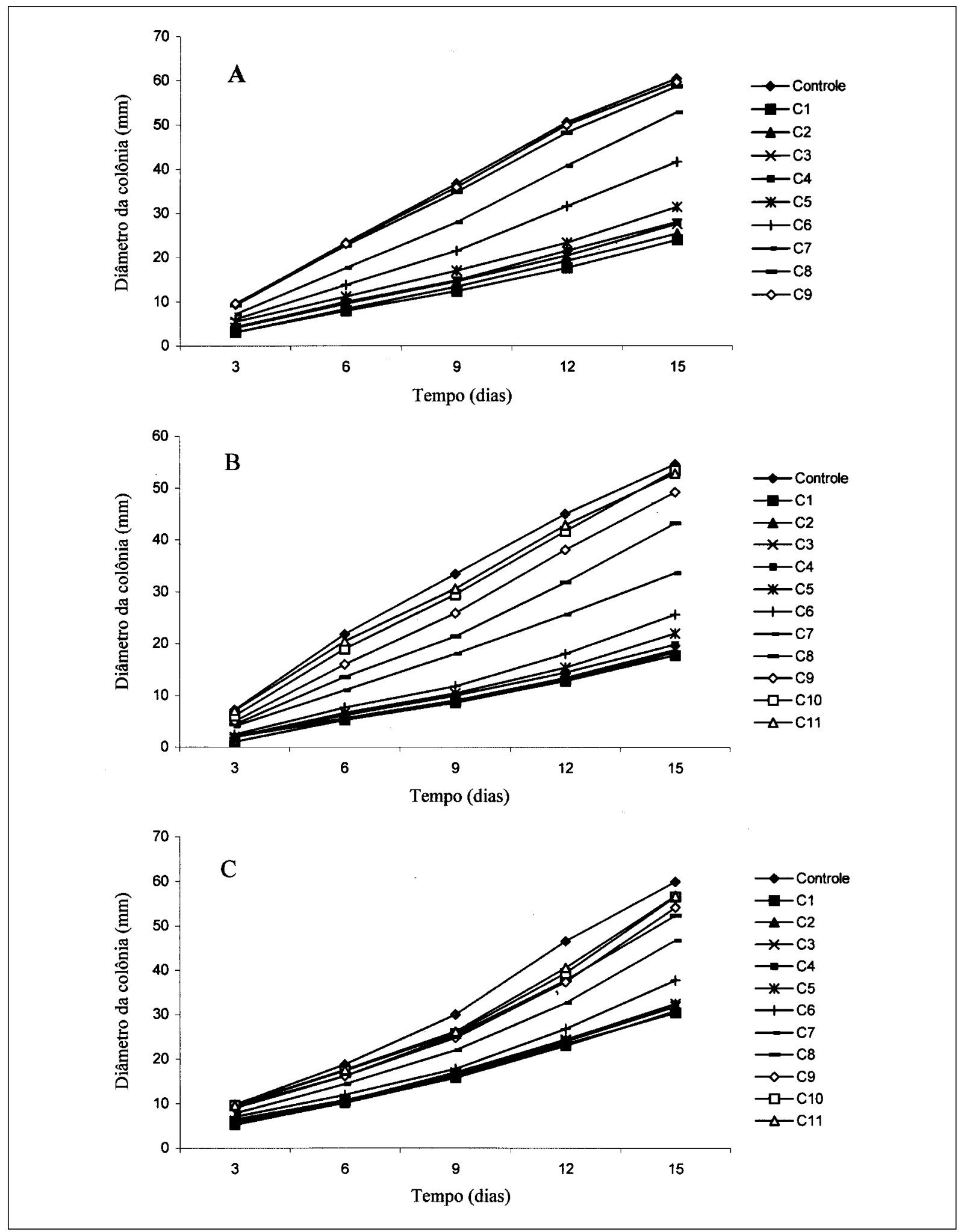

Figura 1 - Crescimento radial das colônias de Metarhizium anisopliae (A), Beauveria bassiana (B) e Paecilomyces farinosus (C) em meio BDA contendo diferentes dose de óleo de nim. Controle: meio isento de óleo de nim; C1: meio contendo 5\% de óleo de nim; C2 a C11: concentrações correspondentes a 1/2, 1/4, 1/8, 1/16, 1/32, 1/64, 1/128, 1/256, 1/512, 1/1024, da C1.

Ciência Rural, v.34, n.6, nov-dez, 2004. 
Tabela 1 - Esporulação de Beauveria bassiana, Paecilomyces farinosus e Metarhizium anisopliae em meios contendo diferentes concentrações de óleo de nim.

\begin{tabular}{lccc}
\hline & \multicolumn{2}{c}{$\mathrm{N}^{\mathrm{o}}$ de esporos $\left(\right.$ x $\left.10^{8} \mathrm{con} / \mathrm{mm}^{2}\right)$} \\
\cline { 2 - 4 } Tratamento & B. bassiana & P. farinosus & M. anisopliae \\
\cline { 2 - 4 } Controle & $7,66 \mathrm{a}$ & $17,66 \mathrm{a}$ & $47,69 \mathrm{a}$ \\
C1 & $0,26 \mathrm{~g}$ & $5,44 \mathrm{f}$ & $6,85 \mathrm{e}$ \\
C2 & $0,29 \mathrm{~g}$ & $8,53 \mathrm{e}$ & $7,44 \mathrm{e}$ \\
C3 & $0,43 \mathrm{fg}$ & $8,9 \mathrm{de}$ & $8,52 \mathrm{e}$ \\
C4 & $0,65 \mathrm{fg}$ & $8,91 \mathrm{de}$ & $8,45 \mathrm{e}$ \\
C5 & $0,87 \mathrm{ef}$ & $9,22 \mathrm{de}$ & $11,45 \mathrm{e}$ \\
C6 & $1,36 \mathrm{e}$ & $10,18 \mathrm{~cd}$ & $17,28 \mathrm{~d}$ \\
C7 & $2,61 \mathrm{~d}$ & $11,12 \mathrm{bc}$ & $29,01 \mathrm{c}$ \\
C8 & $5,22 \mathrm{c}$ & $11,13 \mathrm{bc}$ & $37,35 \mathrm{~b}$ \\
C9 & $6,51 \mathrm{~b}$ & $11,25 \mathrm{bc}$ & $40,24 \mathrm{~b}$ \\
C10 & $6,70 \mathrm{~b}$ & $11,96 \mathrm{~b}$ & \\
C11 & $7,48 \mathrm{a}$ & $12,25 \mathrm{~b}$ & \\
\hline F & 90,52 & 949,55 & 194,57 \\
CV (\%) & 5,05 & 5,22 & 9,09 \\
\hline
\end{tabular}

Médias não seguidas de mesma letra, na coluna, diferenciam estatisticamente entre si pelo teste de Tukey a $5 \%$ de probabilidade de erro. Controle: meio isento de óleo de nim; $\mathrm{C} 1$ : meio contendo 5\% de óleo de nim; C2 a C11: doses correspondentes a $1 / 2,1 / 4,1 / 8,1 / 16,1 / 32,1 / 64,1 / 128,1 / 256$, $1 / 512,1 / 1024$, da C1.

Tabela 2 - Viabilidade de conídios de Beauveria bassiana, Paecilomyces farinosus e Metarhizium anisopliae após cultivo dos fungos em BDA contendo diferentes concentrações de óleo de nim.

\begin{tabular}{lccc}
\hline \multirow{3}{*}{ Tratamento } & \multicolumn{3}{c}{ Viabilidade (\%) } \\
\cline { 2 - 4 } & B. bassiana & P. farinosus & M. anisopliae \\
\hline Controle & $98,67 \mathrm{a}$ & $98,44 \mathrm{a}$ & $98,22 \mathrm{a}$ \\
C1 & $97,55 \mathrm{a}$ & $98,00 \mathrm{a}$ & $97,81 \mathrm{a}$ \\
C2 & $97,78 \mathrm{a}$ & $98,44 \mathrm{a}$ & $97,55 \mathrm{a}$ \\
C3 & $98,00 \mathrm{a}$ & $98,00 \mathrm{a}$ & $98,45 \mathrm{a}$ \\
C4 & $98,00 \mathrm{a}$ & $98,22 \mathrm{a}$ & $98,00 \mathrm{a}$ \\
C5 & $98,23 \mathrm{a}$ & $98,22 \mathrm{a}$ & $98,45 \mathrm{a}$ \\
C6 & $97,78 \mathrm{a}$ & $98,44 \mathrm{a}$ & $98,22 \mathrm{a}$ \\
C7 & $98,44 \mathrm{a}$ & $98,44 \mathrm{a}$ & $98,22 \mathrm{a}$ \\
C8 & $98,22 \mathrm{a}$ & $98,00 \mathrm{a}$ & $98,45 \mathrm{a}$ \\
C9 & $98,00 \mathrm{a}$ & $98,23 \mathrm{a}$ & $98,22 \mathrm{a}$ \\
C10 & $98,22 \mathrm{a}$ & $97,78 \mathrm{a}$ & \\
C11 & $98,44 \mathrm{a}$ & $98,22 \mathrm{a}$ & 0,54 \\
\hline F & 0,42 & 0,2 & 0,71 \\
CV $(\%)$ & 0,87 & 0,88 & \\
\hline
\end{tabular}

Médias não seguidas de mesma letra, na coluna, diferenciam estatisticamente entre si pelo teste de Tukey a 5\% de probabilidade de erro. Controle: meio isento de óleo de nim; $\mathrm{C} 1$ : meio contendo 5\% de óleo de nim; C2 a C11: doses correspondentes a 1/2,1/4,1/8,1/16,1/32,1/64, 1/128, 1/256, 1/512, 1/1024, da C1. quando os conídios foram inoculados em meio contendo as diferentes concentrações do óleo (Tabela 3). Estes resultados demonstram que o óleo de nim não tem efeito na viabilidade dos fungos. Do ponto de vista do uso associado dos fungos com o óleo de nim, no controle biológico de insetos, este resultado é importante, pois mostra o potencial de utilização conjunta de esporos do fungo veiculados em solução aquosa (ou semelhante) de óleo de nim, sem que haja redução da germinação dos esporos.

Respostas variando entre inibitórias, estimulantes ou neutras, têm sido demonstradas para formulações de herbicidas (GARDNER \& STOREY, 1985), fungicidas (KELLER et al., 1993), inseticidas (ANDERSON \& ROBERTS, 1983; AGUDA et al., 1984) e carrapaticidas (PAIÃO, 2000), em estudos de compatibilidade com fungos entomopatogênicos. Contudo, trabalhos que avaliam o efeito de extratos vegetais sobre estes fungos são bastante escassos na literatura.

Segundo QUINTELA et al. (2002), o crescimento de B. bassiana não foi afetado pelo óleo de nim puro (sem emulsificante) oriundo da República Dominicana. O óleo emulsificado (na concentração de 5\%) e o óleo Dalneem (nas concentrações de 0,5,

Tabela 3 - Viabilidade de conídios de Beauveria bassiana Paecilomyces farinosus e Metarhizium anisopliae inoculados em BDA contendo diferentes concentrações de óleo de nim.

\begin{tabular}{lccc}
\hline & \multicolumn{3}{c}{ Viabilidade (\%) } \\
\cline { 2 - 4 } Tratamento & B. bassiana & P. farinosus & M. anisopliae \\
\cline { 2 - 4 } Controle & $98,67 \mathrm{a}$ & $98,67 \mathrm{a}$ & $98,67 \mathrm{a}$ \\
C1 & $98,22 \mathrm{a}$ & $97,87 \mathrm{a}$ & $97,78 \mathrm{a}$ \\
C2 & $98,22 \mathrm{a}$ & $98,23 \mathrm{a}$ & $98,00 \mathrm{a}$ \\
C3 & $98,22 \mathrm{a}$ & $97,78 \mathrm{a}$ & $98,68 \mathrm{a}$ \\
C4 & $98,00 \mathrm{a}$ & $98,22 \mathrm{a}$ & $98,67 \mathrm{a}$ \\
C5 & $98,00 \mathrm{a}$ & $98,00 \mathrm{a}$ & $98,67 \mathrm{a}$ \\
C6 & $98,22 \mathrm{a}$ & $98,44 \mathrm{a}$ & $98,67 \mathrm{a}$ \\
C7 & $98,45 \mathrm{a}$ & $98,00 \mathrm{a}$ & $98,22 \mathrm{a}$ \\
C8 & $98,45 \mathrm{a}$ & $98,89 \mathrm{a}$ & $98,44 \mathrm{a}$ \\
C9 & $97,78 \mathrm{a}$ & $98,00 \mathrm{a}$ & $98,45 \mathrm{a}$ \\
C10 & $98,67 \mathrm{a}$ & $98,45 \mathrm{a}$ & \\
C11 & $98,22 \mathrm{a}$ & $98,22 \mathrm{a}$ & 0,62 \\
\hline F & 0,32 & 0,44 & 0,72 \\
CV (\%) & 0,82 & 0,91 & \\
\hline
\end{tabular}

Médias não seguidas de mesma letra, na coluna, diferenciam estatisticamente entre si pelo teste de Tukey a $5 \%$ de probabilidade de erro. Controle: meio isento de óleo de nim; $\mathrm{C} 1$ meio contendo 5\% de óleo de nim; C2 a C11 doses correspondentes a $1 / 2,1 / 4,1 / 8,1 / 16,1 / 32,1 / 64,1 / 128,1 / 256$, $1 / 512,1 / 1024$, a C1.

Ciência Rural, v.34, n.6, nov-dez, 2004. 
1,0 e 2,0\%) reduziram o crescimento, mas este último óleo, na concentração de $0,25 \%$, aumentou em $7,25 \%$ o crescimento do fungo. A produção de conídios obtida nas concentrações de 1,0 e $2,0 \%$ não diferiu da testemunha. Todas as concentrações do extrato comercial de folhas de Nim reduziram o crescimento micelial, mas as concentrações de 5 e $10 \%$ não afetaram a esporulação do fungo.

O óleo de nim usado neste trabalho reduziu o crescimento dos fungos e afetou a esporulação. Estes resultados coincidem com os obtidos pelos últimos autores com as concentrações mais elevadas do óleo emulsificado e Dalneem, que também reduziram o crescimento, mas divergem quanto à esporulação, já que a mesma não foi afetada pelo Dalneem.

M. anisopliae mostrou-se menos sensível à ação do óleo de Nim, do que B. bassiana. Resultado semelhante foi obtido por GUPTA et al. (2002) que verificaram, em testes de compatibilidade in vitro, que M. anisopliae se mostrou bem menos susceptível à ação da azadiractina do que $\boldsymbol{B}$. bassiana. OLSON \& OETTING (1999) observaram que a ação de $\boldsymbol{B}$. bassiana sobre larvas de Galleria mellonella reduziu em 50\%, após aplicação de azadiractina.

\section{CONCLUSÃO}

O óleo de nim afeta o crescimento e a esporulação de $\boldsymbol{M}$. anisopliae, B. bassiana e $\boldsymbol{P}$. farinosus, mas não afeta a viabilidade dos conídios destes fungos, sendo que $\boldsymbol{M}$. anisopliae é menos sensível a ação do óleo de nim do que os demais fungos.

\section{FONTES DE AQUISIÇÃO}

${ }^{1}$ Hélcio de Abreu Júnior. NIM do Brasil. Rua Clóvis Bevilaqua, 550, D-32, CEP 13075-040, Jardim Guanabara, Campinas, S.P. e-mail: nim.br@uol.com.br

\section{REFERÊNCIAS BIBLIOGRÁFICAS}

AGUDA, R.M. et al. Inhibitory effects of insecticides on entomogenous fungi Metharhizium anisopliae and Beauveria bassiana. International Rice Research Newsletter, Manila, v.9, n.6, p.16-17, 1984.

AMADIOHA, A. C. Controlling rice blast in vitro and in vivo with extracts Azadirachta indica. Crop Protection, Oxford, v. 19 , n.5, p.287-290, 2000.

ANDERSON, T.E.; ROBERTS, D.W. Compatibility of Beauveria bassiana isolates with insecticide formulations used in Colorado potato beetle (Coleoptera: Chrysomelidae) control. Journal of Economic Entomology, Lanham, v.76, p.1437-1441, 1983.

BAKER, F.J. Manual de técnica bacteriológica. Saragoza:
Acribia, 1970. 510p.

BUTTERWORTH, J.H.; MORGAN, E.D. Isolation of a substance that suppresses feeding in locusts. Journal of the Chemical Society, Chemical Communication, London, v.35, n.1, p.23-24, 1968.

CHATTOPADHYAY, C. Yield loss attributable to Alternaria bligh of sunflower (Helianthus annuus L.) in India and some potentially effective control measures. International Journal of Pest Management, London, v.45, n.1, p.15-21, 1999.

DIXON, W.J.; MASSEY JR., F.J. Introduction to statistical analysis. 3.ed. Tokyo : McGrawHill Kogakusha, 1969. 638p.

GARDNER, W.A.; STOREY, G.K. Sensitivity of Beauveria bassiana to selected herbicides. Journal of Economic Entomology, Lanham, v.78, n.6, p.1275-1279, 1985.

GUPTA, R.B.L. et al. Compatibility of two entomofungi, Metarhizium anisopliae and Beauveria bassiana with certain fungicides, insecticides and organic manures. Indian Journal of Entomology, New Delhi, v.64, n.1, p.48-52, 2002.

KELLER, S. et al. Influence of fungicides on ins ect pathogenic fungus Beauveria brongniartii (Sacc.) Petch. Anzeiger fuer Schaedlingskunde Pflanzenschutz Umweltschutz, Hamburg, v.66, n.6, p.108-114, 1993.

LANDA, Z.; BOHATA, A. Compatibility of entomogenous fungus Paecilomyces fumosoroseus whith natural insecticides based on azadirachtin and neem oil. Collection of Scientific Papers - Series for Crop Sciences, Ceske Budejovice, v.16, n.2, p.99-106, 1999.

LOURENÇÃO, A.L. et al. Controle de Sitophilus zeamais em Milho com Beauveria bassiana, Metarhizium anisopliae e Pirimifos Metil. Ecossistema, Pinhal, v.18, p.69-74, 1993.

OLSON, D.L.; OETTING, R.D. Compatibility of insect growth regulators and Beauveria bassiana (Balsamo) Vuillemin in controlling green peach aphid (Homoptera: Aphididae) on greenhouse chrysanthemums. Journal of Entomological Science, Tifton, v.34, n.3, p.186-294, 1999.

PAIÃO, J.C.V. Compatibilidade dos fungos Beauveria bassiana e Metarhizium anisopliae com carrapaticidas químicos utilizados no controle de Boophilus microplus (Acari: Ixodidae). 2000. 55f. Dissertação (Mestrado em Microbiologia) - Programa de Pós-graduação em Microbiologia, Universidade Estadual Paulista.

PRAMILA GUPTA, M. et al. Studies on compatibility of white muscardine fungus Beauveria bassiana with some neem products. Indian Phytopathology, New Delhi, v.52, n.3, p.278280, 1999.

QUINTELA, E.D. et al. Controle de pragas do feijoeiro com o nim indiano e outras plantas. In: BOIÇA JR., A.L. et al. V Curso sobre produção e utilização do Nim (Azadirachta indica) na agropecuária. Jaboticabal : Faculdade de Ciências Agrárias e Veterinárias, 2002. p.1-10

SHASHI, S. et al. Compatibility of Beauveria brongniartii with pesticides and organic manures. Pesticide Research Journal, Barking, v.10, n.2, p.251-253, 1998.

STEINHAUER, B. Possible ways of using the neem tree to control phytophathogenic fungi. Plant Research and Development, Tubingen, v.50, p.83-92, 1999. 